\title{
The rapid diagnosis of isoniazid and rifampicin resistance in Mycobacterium tuberculosis - a molecular story
}

\author{
F. A. DROBNIEWSKI and S. M. WILSON \\ Mycobacterium Reference Unit, Public Health Laboratory Service, Dulwich Hospital, East Dulwich Grove, \\ London SE22 8QF
}

\begin{abstract}
Isoniazid and rifampicin resistance are assayed phenotypically by the resistance ratio, absolute concentration or proportion methods. Assay methods are often difficult to standardise and the World Health Organization (WHO) Global Programme on Drug Resistance is attempting to produce standardised drug resistance data worldwide. Brothbased methods are faster than solid media systems, and a commercial radiometric system, the Bactec 460 , is arguably the fastest method and permits testing to be completed within 7-14 days; however, this method is expensive and requires disposal of radioactive material. Novel phenotypic methods that utilise mycobacteriophages have shown promise. Other molecular detection systems require knowledge of the genes encoding the drug target (the $\operatorname{inh} A / m a b A, k a t G, o x y R$ and $a h p C$ genes for isoniazid; $r p o B$ for rifampicin) and the mutations producing resistance. These genotypic methods are limited in that not all resistance mechanisms are known, but advanced assays for rifampicin resistance that use gene sequencing, heteroduplex analysis, solid-phase hybridisation or single-strand conformation polymorphism analysis are becoming available.
\end{abstract}

\section{Introduction}

According to current estimates of the World Health Organization (WHO), 8 million cases of tuberculosis (TB) occur each year, resulting in 3 million deaths [1-3]. Almost one-third of the earth's population, 1.7 billion people, are believed to be infected by the causative organism Mycobacterium tuberculosis [2]. The steady decline in the incidence of TB in high income countries ceased or reversed in the 1980s. However, most TB cases occur in the developing world, where there continues to be a significant increase in incidence. In 1993-1995, the bulk of cases were found in the South-East Asian (42\%), Western Pacific (23.5\%) and African (14.5\%) regions, compared to Europe $(8.1 \%)$, the Americans $(6.9 \%)$ and the Eastern Mediterranean $(5.0 \%)$ [4]. Factors such as poverty, homelessness, civil disturbance, poorly functioning National TB Programmes (NTP) and the dismantling of public health infrastructure have contributed significantly to the worsening situation [3-5]. The association of TB with infection by human immunodeficiency virus (HIV) has proved to be a major new factor in the increase of

Received 12 Aug. 1997; accepted 26 Aug. 1997.

Corresponding author: Dr F. A. Drobniewski. clinical TB, particularly in the developing world, where even model programmes such as those supported by the International Union Against Tuberculosis and Lung Disease (IUATLD) have faltered [4-6].

\section{Drug resistance}

The real magnitude of drug resistance world-wide in $M$. tuberculosis is not known, as it is difficult to ensure that representative populations have been sampled and there are often severe limitations on the infrastructure and quality control mechanisms needed to perform culture and drug susceptibility testing in many parts of the world. In a recent review of drug resistance surveys, Cohn et al. [7] concluded that overall primary resistance (new cases with no previous treatment or no report of previous treatment) rates were $0-16.9 \%$ for isoniazid, $0-3 \%$ for rifampicin resistance, $0-4.2 \%$ for ethamubutol resistance, and $0.1-23.5 \%$ for streptomycin resistance; acquired resistance rates (resistance in patients described as treatment failures - i.e., those patients who are re-treatment cases or who have had treatment previously) are significantly higher. In some cities where treatment completion rates are particularly poor, resistance may be much higher. 
Multi-drug resistant $M$. tuberculosis (MDRTB) strains, defined as strains that are resistant to isoniazid and rifampicin, cause most concern because morbidity and mortality rates are higher than in TB caused by drugsensitive strains, especially in patients with HIV infection. Second- or third-line drugs must be used which have greater side-effects and, as treatment is prolonged, the overall cost of treatment and toxicity are increased. Patients remain infectious for longer periods, which increases the risk of transmission. Several outbreaks of MDRTB in the USA, most notably in the New York penal and hospital systems, and also in Europe, have aroused considerable alarm. Mortality rates in patients co-infected with MDRTB and HIV can be as high as $80-90 \%$, although prompt diagnosis and early aggressive therapy can reduce this figure [5]. The rates of MDRTB were relatively low in most countries where representative studies have been conducted, with primary resistance rates of $0-10.8 \%$, but with rates for acquired resistance of $0-48 \%$ [7].

Currently, the WHO is co-ordinating a Global Surveillance Programme of Drug Resistance in TB. Measuring the drug susceptibility of isolates has three main purposes. Firstly, it confirms the efficacy of an empirical treatment regimen in a given individual, or permits the tailoring of treatment in the case of drug resistance. Secondly, it enables medical and public health services to plan appropriate empirical regimens to address problems of emerging drug resistance. Thirdly, it is a useful crude indicator of the efficacy of a treatment programme. For example, poor case handling and compliance lead to increasing levels of acquired drug resistance.

Within the UK, identification of mycobacteria and drug susceptibility testing is organised as indicated in Fig. 1 and is described in more detail later.

Drug resistance is measured currently with a variety of liquid (e.g., Middlebrook broth $7 \mathrm{H} 9$ or $7 \mathrm{H} 12$ ) or solid (e.g., Lowenstein-Jensen, Middlebrook $7 \mathrm{H} 10$ or $7 \mathrm{H} 11$ ) media by one of three basic methods: the resistance ratio, the absolute concentration (or MIC), or the proportion method [8-10]. Broth-based methods are faster than solid media systems and, currently, a commercial radiometric system permits antimicrobial drug susceptibility testing to be performed by the

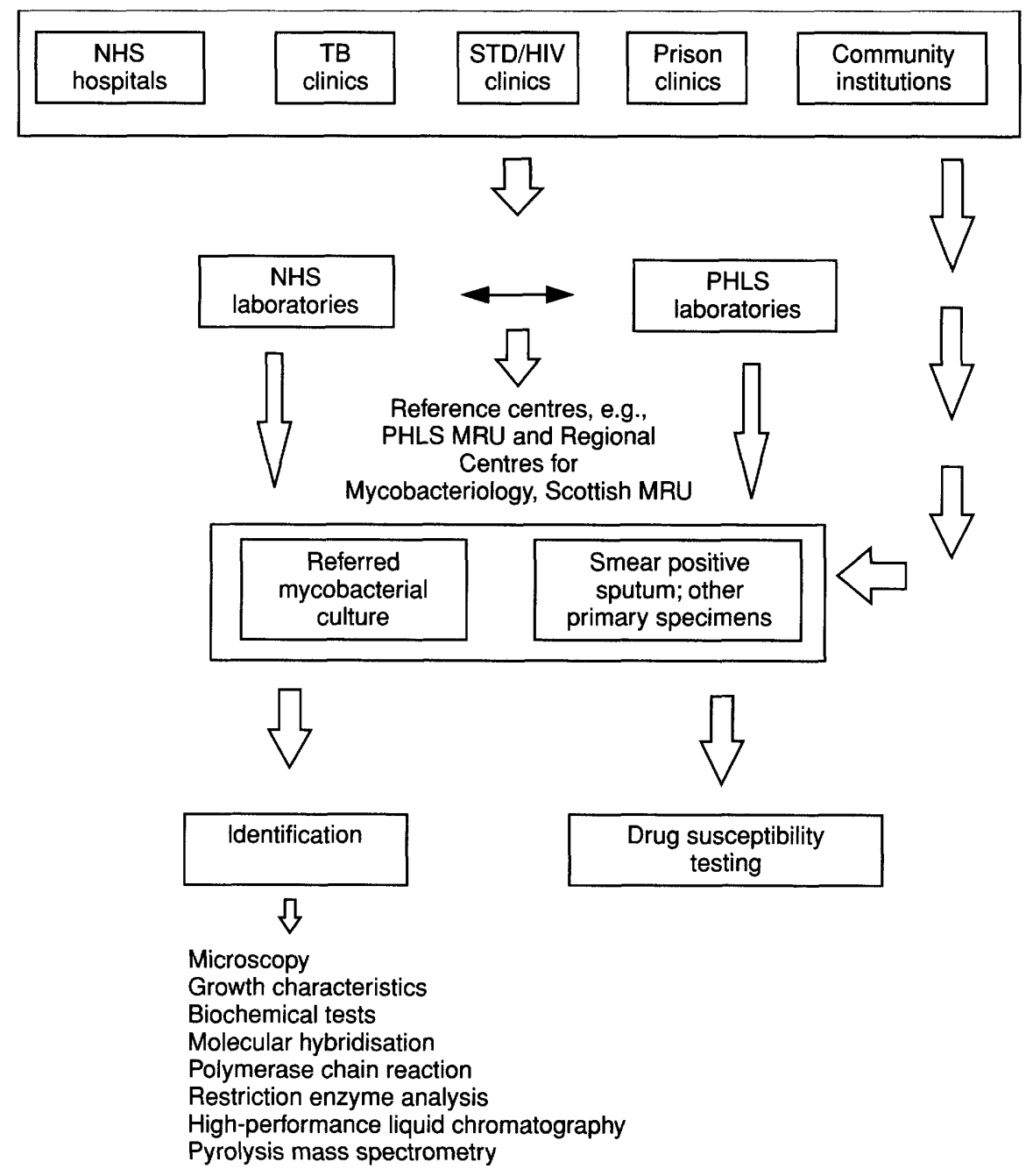

Fig. 1. Flow diagram showing the organisational structure for the identification and drug susceptibility testing of mycobacteria in Great Britain. 
proportional method within 7-14 days following initial growth of the organism. All methods are affected to different extents by problems relating to variations in the inoculum size and the stability of antimicrobial agents.

Assays involving the use of radiometric agents are becoming increasingly difficult in the context of clinical rather than research laboratories because of greater restrictions on their use and the cost of radioactive disposal. Nevertheless, culture-based methods are likely to remain the mainstay of drug susceptibility testing for the complete current range of drugs for some time to come, particularly as automated non-radiometric rapid culture systems are becoming available more widely. The increased automation of these systems will reduce the handson time needed to monitor growth.

In some cases where there is a suspicion that the patient may harbour a drug-resistant strain, e.g., if the patient is a contact of a known resistant case or has relapsed from a TB infection treated previously, there is a need to perform drug susceptibility tests rapidly. Rapid determination of susceptibility would establish an effective treatment regimen and indicate the degree and extent of patient isolation or isolation precautions required. Rapid susceptibility testing can be achieved with novel molecular assays which have the potential for defining drug resistance for the principal first-line drugs such as isoniazid and rifampicin within $24-48 \mathrm{~h}$ of obtaining a culture and, more importantly, in primary specimens with little or no prior culture. A scenario in which preliminary culture of primary specimens for $48-72 \mathrm{~h}$ is coupled with novel molecular drug susceptibility testing technologies may provide the optimal balance between sensitivity, specificity and turnaround time, particularly in specimens such as cerebrospinal fluid (CSF) or pleural fluid (PF) where the number of organisms is likely to be low and diagnosis is difficult.

Novel drug assays can be divided into phenotypic or genotypic systems; in the former, the outcome, such as death of the bacterium, is measured without any previous knowledge of the underlying resistance mechanism, whereas in the latter, the drug target and nature of the gene mutations are known (Table 1). Fig. 2 indicates the types and range of genotypic and phenotypic assays that are becoming available in research and reference centres.

\section{Genotypic methods}

Genotypic assays combine three main steps: (a) sample preparation to release DNA from mycobacterial cells; (b) amplification of the relevant part of the gene, usually by the polymerase chain reaction (PCR); and (c) detection of the mutation in comparison with the equivalent region of a wild-type strain.

Table 1 indicates the current known targets of the major drugs currently used in treatment of TB and the corresponding mutations in the genes that cause resistance. For example, mutations within an 81-bp region (codons 507-533) of the rpoB gene encoding the $\beta$ chain of the DNA-dependent RNA polymerase confer resistance in $c$. $95 \%$ of all clinical isolates examined [11-13]. More than 35 mutations within this region have been described [14], but two mutations (His $526 \rightarrow$ Tyr and Ser $531 \rightarrow$ Leu) account for two-thirds of rifampicin resistance [14, 15]. Genotypic assays that detect mutations within this region are, therefore, predictive of clinical drug resistance. However, the situation is much more complex if isoniazid resistance is considered. Mutations in at least four genes or gene complexes are known to be involved in resistance, including kat $G$ which encodes the catalase-peroxidase enzyme, inh $A$ / $m a b A$ which is involved in fatty acid biosynthesis, $\operatorname{ahpC}$ which encodes the alkyl hydroperoxide reductase $\mathrm{C}$, and oxyR which is the oxidative stress regulator [16-24]. To detect isoniazid resistance would require systems that were capable of detecting mutations at multiple sites simultaneously, and such systems are able currently to predict isoniazid resistance only in c. $85 \%$ of cultures [24]. A similar situation exists for ciprofloxacin and streptomycin resistance which can be predicted with $c$. $75 \%$ and

Table 1. Gene targets for anti-tuberculosis drugs and mutations associated with drug resistance

\begin{tabular}{|c|c|c|c|}
\hline Drug & Probable targets & $\begin{array}{l}\text { Mutation in genes conferring } \\
\text { resistance }\end{array}$ & Function of gene \\
\hline Rifampicin & RNA synthesis & rpoB & DNA-dependent RNA polymerase ( $\beta$ subunit) \\
\hline \multirow[t]{4}{*}{ Isoniazid } & Mycolic acid & kat $G$ & Catalase/peroxidase \\
\hline & biosynthesis & $\operatorname{inh} A / m a b A$ & Fatty acid biosynthesis \\
\hline & & $a h p C$ & Alkyl hydroperoxide $\mathrm{C}$ reductase \\
\hline & & $O x y R$ & Oxidative stress regulator \\
\hline Pyrazinamide & Pyrazinamide (Pzase) $+?$ & pncA & Pzase nicotinamidase; loss of Pzase gives resistance \\
\hline \multirow[t]{2}{*}{ Streptomycin } & Protein synthesis & $r r s$ & $16 \mathrm{~S}$ rRNA \\
\hline & & $r p s L$ & Ribosomal protein $\mathrm{S} 12$ \\
\hline Ethambutol & Cell wall synthesis & $e m b A, B, C$ & Lipoarabinomannan and arabinogalactan synthesis \\
\hline Ethionamide & Cell wall synthesis & $\operatorname{inh} A+?$ & Cross-resistance associated with inhA mutations \\
\hline Ciprofloxacin & DNA synthesis & gyr $A$, gyr $B$, nor & DNA gyrase subunit A and B (principally) \\
\hline
\end{tabular}

PCR, polymerase chain reaction; SSCP, single strand conformational polymorphism. 


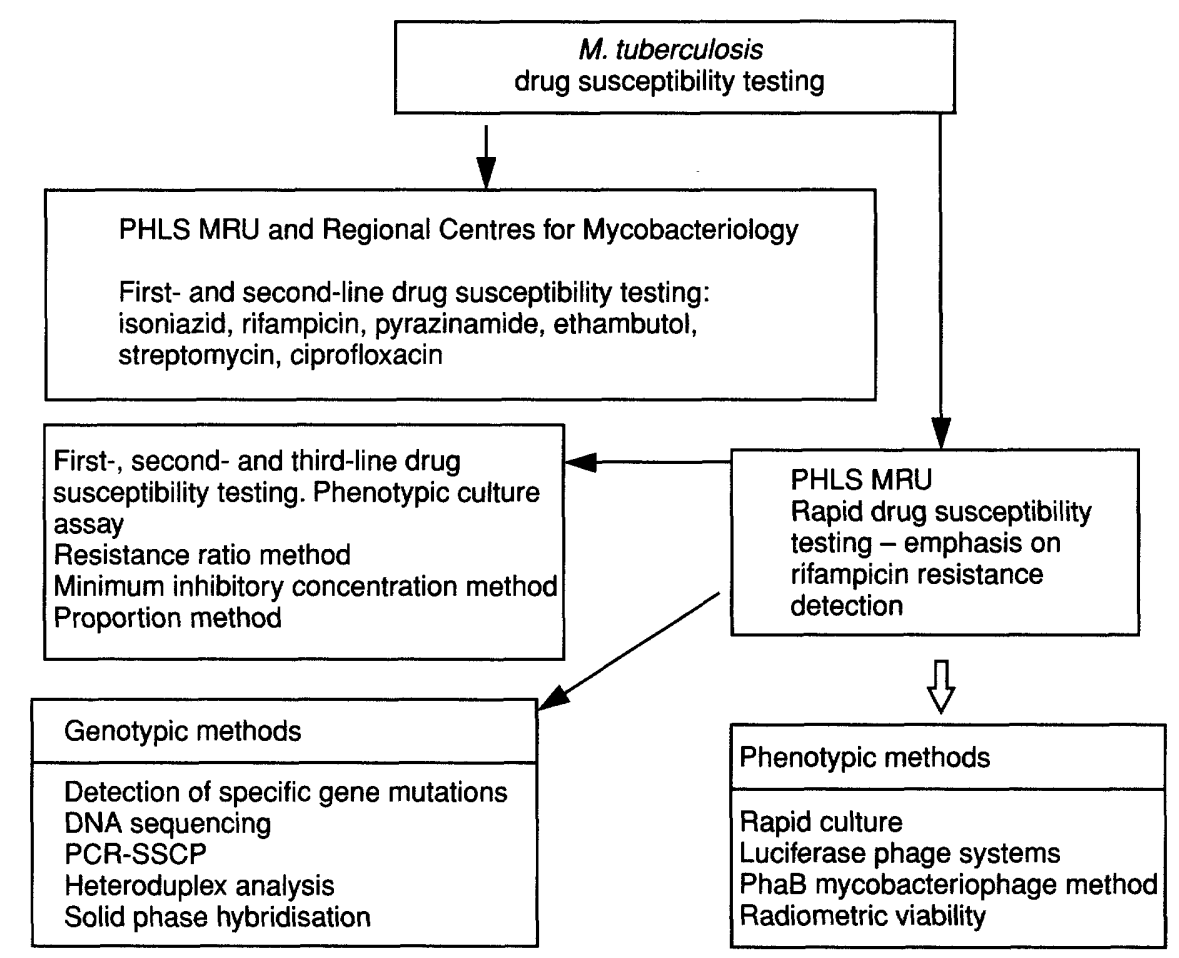

Fig. 2. Drug susceptibility testing within the PHLS and the methods used or described for phenotypic and genotypic detection of resistance.

$60 \%$ certainty, respectively, by genotypic methodologies [25-29].

Detection of resistance to rifampicin and isoniazid is essential for recognition of MDRTB strains responsible for nosocomial and other institutional outbreaks, particularly in HIV-positive individuals, where they have been associated with a high mortality. The value of genotypic methodologies is that they have the potential for direct application to clinical samples. Further work is continuing at research centres including the UK Public Health Laboratory Service (PHLS) Mycobacterium Reference Unit (MRU) to extend these assays to primary clinical specimens. Methods which have been used for genotypic susceptibility testing include DNA sequencing, heteroduplex analysis, solid-phase hybridisation analysis and PCR single-strand conformation polymorphism (SSCP) analysis.

\section{DNA sequencing}

DNA sequencing remains the gold standard, as all mutations will be detected. In the case of rifampicin, the presence of mutations appears to be fully predictive of resistance; however, this is not necessarily the case with other genes associated with drug resistance, as silent mutations that do not lead to any significant change in phenotype also occur. For example, a common site for mutations within the C-terminal region of the kat $G$ gene has little effect on the activity of the catalase enzyme $[14,19,20]$. Manual DNA sequencing requires a high level of technical competence and employs a radiolabel which must be safely disposed of, making the technique unsuitable for busy clinical laboratories receiving hundreds of specimens. Automated sequencers that use fluorescent-based chemistry can provide accurate sequence data within $24-48 \mathrm{~h}$, but are costly, relatively expensive to maintain, and require a high degree of operator expertise for consistently reliable data. However, in addition to detecting mutations in genes involved with isoniazid and rifampicin resistance, automated sequencing has been used to detect mutations in the gyr A, rrs and $r p s L$ genes associated with ciprofloxacin and streptomycin resistance, respectively [19, 25, 26, 29].

\section{Heteroduplex analysis}

In this method, amplified DNA from test organisms and reference drug-sensitive strains is mixed, denatured and cooled to produce double-stranded hybrid DNAs through complementary base pairing. In regions where mutations occur, e.g., in a drug-resistant test strain, there will be a mismatch of the two strands and the resulting heteroduplex will run with a different mobility to the homoduplex (where the test strain has no mutations) on a denaturing electrophoresis gel system. This method has been applied to the detection of rifampicin resistance [30].

\section{Solid-phase hybridisation analysis}

Mutations can be detected by solid-phase hybridisation analysis. For example, in order to detect any mutations which may encode rifampicin resistance, a series of overlapping probes that are complementary to the 81bp region of the $r p o B$ gene are immobilised on to a 
solid membrane support. In addition, probes complementary to the most common rifampicin-resistance mutations can be included. The $r p o B$ gene fragment is amplified from a test strain by PCR and then reverse hybridised to the membrane containing the probes [15, 31]. Failure of this amplified DNA to bind to any of the sensitive sequence-specific probes indicates mutations that may encode resistance. Conversely, if the product hybridises to any of the mutation-specific probes, the mutation can be characterised directly. Such a system, the Line Probe Assay (Innogenetics, Ghent, Belgium) has been used with some success $[15,32]$. This assay includes probes for four mutations, including the two commonest (His $536 \rightarrow$ Tyr and Ser $531 \rightarrow$ Leu), but the ability to identify less frequent mutations correctly is unknown, i.e., the assay might be unable to detect certain insertional mutations within this region. In the USA, the assay was used for the rapid identification of rifampicin resistance in a collection of 51 rifampicinresistant cultures. Nine distinct $r p o B$ mutations were detected and concordance with standard phenotypic rifampicin susceptibility testing results was $90.2 \%$ [15]. At the PHLS MRU this assay has been used prospectively on a collection of 51 cultures with promising results. Thus 22 of 23 culture-defined rifampicin-resistant isolates were found to be resistant by the Line Probe Assay ( 24 rather than 23 were defined as sensitive), and five cultures were identified correctly as not being members of the $M$. tuberculosis complex (unpublished results). A prospective study of the diagnosis of resistance in primary specimens is in progress.

\section{$P C R$ single-strand conformation polymorphism (PCR-SSCP) analysis}

Single-stranded DNA folds into a complex tertiary structure whose shape is determined by the DNA sequence. If any two single strands of DNA differ by one or more bases, i.e., a mutation is present, they will fold into structures with different mobility on a polyacrylamide gel. This is termed a single-strand conformation polymorphism (SSCP). In conjunction with PCR used to amplify the region of the gene of interest, SSCP analysis has been used to detect rifampicin, ciprofloxacin, isoniazid and streptomycin resistance $[11,20,24,33]$. Normally the method uses a radioactive detection system, but silver staining has been described recently as an alternative and sensitive method. Automation of the process permits a higher throughput of specimens, but increases the cost as an automated sequencer is required to detect migration of the bands.

\section{Phenotypic methods}

Culture-based phenotypic methods, in which the survival of a test strain in comparison with a drugsensitive control is assayed on a solid or liquid medium, continues to form the mainstay of drug susceptibility testing in both developed and developing countries. Improvements in the rapidity of testing have been developed, e.g., by use of the proportion method in rapid radiometric Bactec liquid culture systems [34] in which a mycobacterial culture is inoculated into drug-containing Bactec vials and a control vial is inoculated with a 100-fold dilution of the inoculum. Equivalent or greater growth in the drug-containing test vial compared to the control vial indicates that $\geqslant 1 \%$ of the population of bacilli are drug-resistant and that the drug is unlikely to be effective. Further work has continued to develop systems for non-radiometric determination, e.g., the MGIT system [35, 36], although such systems are more expensive than current methods that use solid media. To speed up the procedure, direct drug susceptibility testing can be performed on those samples that show acid-fast bacilli in smears [37].

Recently, two novel mycobacteriophage (viruses that are specific for mycobacteria) assays have been developed. Jacobs et al. [38] demonstrated that drug susceptibility could be measured with genetically modified mycobacteriophages containing the $l u x$ or luciferase gene. Drug-resistant mycobacteria growing in the presence of these drugs are able to express luciferase, which catalyses the reaction of luciferin with ATP to generate photons of light that can be detected in a luminometer, while mycobacteria sensitive to the drug die, no ATP is produced, and there is a cessation of light production. This approach has the advantage that no knowledge of the underlying genetic basis of resistance is needed, but it requires expensive equipment, which may make the technique unsuitable for the developing world where much of the disease burden occurs. An alternative approach, the PhaB assay (phage amplified biologically), involves mycobacteriophage, but without the need for expensive luminometers [39]. This approach utilises skills and resources that are available readily within the diagnostic laboratory. The $\mathrm{PhaB}$ assay can be used for diagnosis of MTB in patient samples, and also for direct drug susceptibility testing of organisms isolated previously by conventional culture. The concept of the method is simple (Fig. 3). In the first step, any viable mycobacteria contained in a test sample are infected with mycobacteriophage. Subsequently, any mycobacteriophage remaining outside the mycobacteria are inactivated by a specific chemical treatment, whereas those inside mycobacteria are protected. The protected mycobacteriophage then replicate within viable bacilli and, eventually, cause the host mycobacteria to lyse and release a new generation of mycobacteriophage. The released mycobacteriophage are plated on to a lawn of the rapidly growing species $M$. smegmatis. The mycobacteriophages infect and replicate in this related organism and, after an overnight incubation period, are detected as clear areas of lysis or plaques in the turbid growth of the $M$. smegmatis lawn. The 


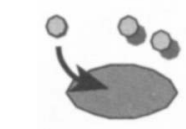

1. Phage infect slowgrowing $M$. tuberculosis
2. Exogenous phage are inactivated. Endogenous phage are protected

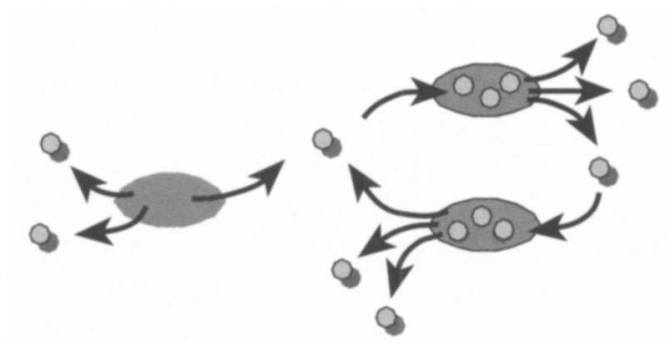

4. The phage progeny rupture the host
5. The released phage are mixed with fast-growing $M$. smegmatis and undergo many cycles of replication and lysis

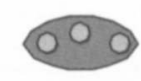

3. The protected phage replicate

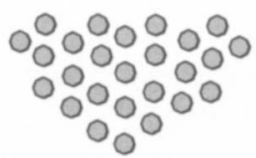

6. The progeny phage are quantified which gives an indication of the number of viable $M$. tuberculosis that were in the original sample

Fig. 3. Diagrammatic representation of the mycobacteriophage PhaB assay.

procedure is potentially rapid and simple to perform, taking as little as $48 \mathrm{~h}$ compared to the several weeks required for conventional culture. The sensitivity of the assay has been calculated to be as few as 10-100 mycobacteria/ml of sample, which is at least as sensitive as conventional culture. The assay involves the use of simple equipment and, as the assay proceeds, the number of viable infectious MTB particles actually declines, so that unlike the systems described above, the assay actually becomes safer with increasing duration.

In a recent evaluation the assay correctly assigned and identified all eight MDR isolates and the single rifampicin-resistant, isoniazid-sensitive isolate examined. When used to test susceptibility to isoniazid, the PhaB assay identified $15(88.2 \%)$ of 17 isoniazidresistant, rifampicin-sensitive isolates and $17(81 \%)$ of 21 isoniazid-sensitive isolates. The results were obtained in 3-4 days, but recent results suggest that rifampicin testing can be done in as little as 2 days from receipt of a patient sample.

\section{Infrastructure and strategy for drug susceptibility testing}

The current arrangement for identification and drug susceptibility testing of mycobacterial cultures in Great Britain is represented in Fig. 1. Mycobacterial cultures isolated by National Health Service (NHS) and PHLS laboratories are referred on a geographical basis to one of four PHLS Regional Centres for Mycobacteriology (RCM), co-ordinated by the PHLS MRU, which also acts as the Regional Centre for southern England. Over $95 \%$ of mycobacterial cultures in Great Britain are referred to these four centres and to the Scottish MRU. Identification is performed by a variety of methods including microscopy, growth characteristics, biochemical tests, molecular hybridisation systems and, where necessary, PCR-based systems, pyrolysis mass spectrometry and other specialised techniques. Drug susceptibility testing is performed in solid or liquid media with standardised methodology including the resistance ratio, MIC and proportion methods [8, 9, 37, 40]. Mycobacterial identification and drug susceptibility methods are quality-controlled rigorously to form an integrated mycobacterial reference network. All RCMs receive primary specimens from local hospitals and other institutions in their region, and the PHLS MRU offers a national service of specialised molecular investigations for diagnosis, drug susceptibility testing and molecular epidemiological typing, including the investigation of outbreaks and surveillance of drug resistance.

The mycobacteriology reference network, together with the PHLS Communicable Disease Surveillance Centre (CDSC), forms an integrated tuberculosis programme to provide diagnostic, reference, epidemiological, clinical and research activities at local, regional and national levels. Increasing world-wide and national concern regarding MDRTB, particularly recent nosocomial outbreaks, has resulted in the development of rapid detection methods for rifampicin resistance on a national basis in culture and in primary specimens on a research basis (Fig. 4). Defining rifampicin resistance is predictive of MDRTB, as $85-90 \%$ of rifampicin-resistant isolates in England and Wales are also isoniazid-resistant (unpublished data). Second- and third-line drug susceptibility tests can be performed earlier and stringent infection control measures can be implemented promptly, which may be vital in institutions treating immunocompromised individuals, including those co-infected with HIV. However, currently, the available genetic tests are expensive. Consequently, 


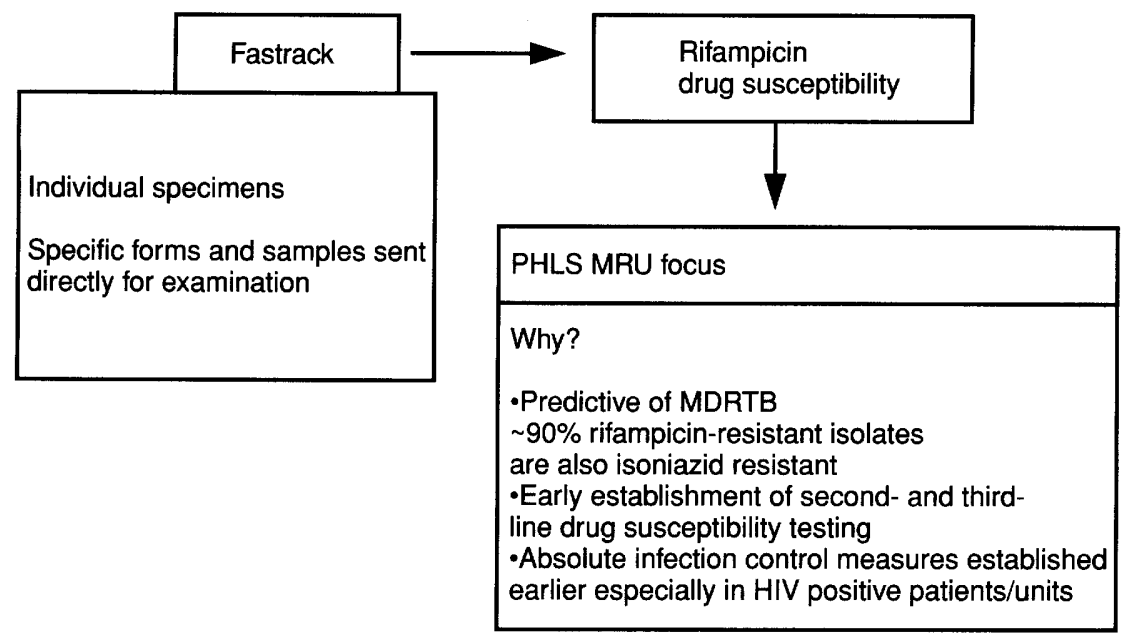

Fig. 4. Rapid diagnosis and drug susceptibility testing: the Fastrack concept.

rapid molecular testing is focused on those samples for which there is a reasonable clinical or epidemiological suspicion of infection with resistant strains. Such samples are currently submitted to the MRU for testing under a Fastrack scheme. The new rapid phenotypic tests promise to be cheaper, but it remains to be seen whether these new approaches can be applied as a routine system for the screening of all clinical samples.

\section{Conclusions}

Underlying basic research to define the mechanisms of drug resistance has been essential to the development of genotypic assays that can be applied in the clinical context. In the case of rifampicin, the presence of mutations within a small region of a single gene are predictive of drug resistance in $M$. tuberculosis (although not in other mycobacterial species). The mechanisms of isoniazid resistance are more complex and the underlying causes of resistance to other antimycobacterial drugs have not been established fully. Genotypic assays are, at present, focused on high-risk specimens, but should be performed in specialist centres where a variety of conventional and rapid culture-based methods (which are likely to remain the mainstay of drug susceptibility tests) can be performed in parallel. New rapid phenotypic approaches, such as the luciferase phage and $\mathrm{PhaB}$ assays, may offer an inexpensive and more comprehensive alternative for the rapid screening of clinical samples and isolates in the future.

\section{References}

1. Dolin PJ, Raviglione MC, Kochi A. Global tuberculosis incidence and mortality during 1990-2000. Bull World Health Organ 1994; 72: 213-220.

2. Sudre P, ten Dam G, Kochi A. Tuberculosis: a global overview of the situation today. Bull World Health Organ 1992; 70: 149-159.

3. Raviglione MC, Snider DE, Kochi A. Global epidemiology of tuberculosis - Morbidity and mortality of a worldwide epidemic. JAMA 1995; 273: 220-226.
4. Drobniewski FA, Pablos-Mendes A, Raviglione M. Epidemiology of tuberculosis in the world today. Semin Respir Crit Care Med 1998 (in press).

5. Drobniewski FA. Is death inevitable with multiresistant TB plus HIV infection? Lancet 1997; 349: 71-72.

6. De Cock KM, Soro B, Coulibaly IM, Lucas SB. Tuberculosis and HIV infection in Sub-Saharan Africa. JAMA 1992; 268: 1581-1587.

7. Cohn DL, Bustreo F, Raviglione MC. Drug-resistant tuberculosis: review of the worldwide situation and the WHO/IUATLD Global Surveillance Project. Clin Infect Dis 1997; 24 (Suppl. 1): S121-S130.

8. Canetti G, Froman S, Grosset $\mathrm{J}$ et al. Mycobacteria: laboratory methods for testing drug sensitivity and resistance. Bull World Health Organ 1963; 29: 565-578.

9. Canetti G, Fox W, Khomenko A et al. Advances in techniques of testing mycobacterial drug sensitivity, and the use of sensitivity tests in tuberculosis control programmes. Bull World Health Organ 1969; 41: 21-43.

10. Vareldzis BP, Grosset J, de Kantor I et al. Drug-resistant tuberculosis: laboratory issues. World Health Organization recommendations. Tuber Lung Dis 1994; 75: 1-7.

11. Telenti A, Imboden $\mathrm{P}$, Marchesi $\mathrm{F}$ et al. Detection of rifampicin-resistance mutations in Mycobacterium tuberculosis. Lancet 1993; 341: 647-650.

12. Kapur V, Li LL, Hamrick MR et al. Rapid Mycobacterium species assignments and unambiguous identification of mutations associated with antimicrobial resistance in Mycobacterium tuberculosis by automated DNA sequencing. Arch Pathol Lab Med 1995; 119: 131-138.

13. Miller LP, Crawford JT, Shinnick TM. The rpoB gene of Mycobacterium tuberculosis. Antimicrob Agents Chemother 1994; 38: 805-811.

14. Musser JM. Antimicrobial agent resistance in mycobacteria: molecular genetic insights. Clin Microbiol Rev 1995; 8: 496-514.

15. Cooksey RC, Morlock GP, Glickman S, Crawford JT. Evaluation of a line probe assay kit for characterization of $r p o B$ mutations in rifampicin-resistant Mycobacterium tuberculosis isolates from New York City. J Clin Microbiol 1997; 35: $1281-1283$.

16. Zhang Y, Heym B, Allen B, Young D, Cole S. The catalaseperoxidase gene and isoniazid resistance of Mycobacterium tuberculosis. Nature 1992; 358: 591-593.

17. Banerjee A, Dubnau E, Quemard A et al. InhA, a gene encoding a target for isoniazid and ethionamide in Mycobacterium tuberculosis. Science 1994; 263: 227-230.

18. Heym B, Zhang Y, Poulet S, Young D, Cole ST. Characterization of the katG gene encoding a catalase-peroxidase required for the isoniazid susceptibility of Mycobacterium tuberculosis. $J$ Bacteriol 1993; 175: 4255-4259.

19. Heym B, Honoré N, Truffot-Pernot $\mathrm{C}$ et al. Implications of multidrug resistance for the future of short-course chemotherapy of tuberculosis: a molecular study. Lancet 1994; 344: 293-298.

20. Heym B, Alzari PM, Honoré N, Cole ST. Missense mutations 
in the catalase-peroxidase gene, katG, are associated with isoniazid resistance in Mycobacterium tuberculosis. Mol Microbiol 1995; 15: 235-245.

21. Wilson TM, de Lisle GW, Collins DM. Effect of inhA and $k a t G$ on isoniazid resistance and virulence of Mycobacterium bovis. Mol Microbiol 1995; 15: 1009-1015.

22. Rouse DA, Li Z, Bai GH, Morris SL. Characterization of the kat $G$ and inhA genes of isoniazid-resistant clinical isolates of Mycobacterium tuberculosis. Antimicrob Agents Chemother 1995; 39: 2472-2477.

23. Morris S, Bai GH, Suffys P, Portillo-Gomez L, Fairchok M, Rouse D. Molecular mechanisms of multiple drug resistance in clinical isolates of Mycobacterium tuberculosis. J Infect Dis 1995; 171: 954-960.

24. Telenti A, Honoré $\mathrm{N}$, Bernasconi $\mathrm{C}$ et al. Genotypic assessment of isoniazid and rifampin resistance in Mycobacterium tuberculosis - a blind study at reference laboratory level. J Clin Microbiol 1997; 35: 719-723.

25. Cooksey RC, Morlock GP, McQueen A, Glickman SE, Crawford JT. Characterization of streptomycin resistance mechanisms among Mycobacterium tuberculosis isolates from patients in New York City. Antimicrob Agents Chemother 1996; 40: 1186-1188.

26. Finken M, Kirschner P, Meier A, Wrede A, Böttger EC. Molecular basis of streptomycin resistance in Mycobacterium tuberculosis: alterations of the ribosomal protein $\mathrm{S} 12$ gene and point mutations within a functional $16 \mathrm{~S}$ ribosomal RNA pseudoknot. Mol Microbiol 1993; 9: 1239-1246.

27. Nair J, Rouse DA, Bai G-H, Morris SL. The rpsL gene and streptomycin resistance in single and multiple drug-resistance strains of Mycobacterium tuberculosis. Mol Microbiol 1993; 10: $521-527$.

28. Meier A, Kirschner P, Bange FC, Vogel U, Bottger EC. Genetic alterations in streptomycin-resistant Mycobacterium tuberculosis: mapping of mutations conferring resistance. Antimicrob Agents Chemother 1994; 38: 228-233.

29. Takiff HE, Salazar L, Guerrero C et al. Cloning and nucleotide sequence of Mycobacterium tuberculosis gyrA and gyrB genes and detection of quinolone resistance mutations. Antimicrob Agents Chemother 1994; 38: 773-780.
30. Williams DL, Waguespack $\mathrm{C}$, Eisenach $\mathrm{K}$ et al. Characterization of rifampicin-resistance in pathogenic mycobacteria. Antimicrob Agents Chemother 1994; 38: 2380-2386.

31. Saiki RK, Walsh PS, Levenson CH, Erlich HA. Genetic analysis of amplified DNA with immobilized sequence-specific oligonucleotide probes. Proc Natl Acad Sci USA 1989; 86: 6230-6234.

32. De Beenhouwer H, Lhiang Z, Jannes G et al. Rapid detection of rifampicin resistance in sputum and biopsy specimens from tuberculosis patients by PCR and line probe assay. Tuber Lung Dis 1995; 76: 425-430.

33. Telenti A, Imboden $P$, Marchesi F, Schmidheini T, Bodmer $T$. Direct, automated detection of rifampicin-resistant Mycobacterium tuberculosis by polymerase chain reaction and singlestrand conformation polymorphism analysis. Antimicrob Agents Chemother 1993; 37: 2054-2058.

34. Middlebrook G, Reggiardo Z, Tigertt WD. Automatable radiometric detection of growth of Mycobacterium tuberculosis in selective media. Am Rev Respir Dis 1977; 115: 1066-1069.

35. Resiner BS, Gatson AM, Woods GL. Evaluation of mycobacteria growth indicator tubes for susceptibility testing of Mycobacterium tuberculosis to isoniazid and rifampicin. Diagn Microbiol Infect Dis 1995; 22: 325-329.

36. Pfyffer GE, Welscher H-M, Kissling $P$ et al. Comparison of the Mycobacteria Growth Indicator Tube (MGIT) with radiometric and solid culture for recovery of acid-fast bacilli. $J$ Clin Microbiol 1997; 35: 364-368.

37. Heifets LB. Drug susceptibility testing. In: Heifets LB (ed) Clinics in laboratory medicine. Philadelphia, Saunders. 1996: 641-656.

38. Jacobs WR, Barletta RG, Udani R et al. Rapid assessment of drug susceptibilities of Mycobacterium tuberculosis by means of luciferase reporter phages. Science 1993; 260: 819-822.

39. Wilson SM, Al-Suwaidi Z, McNerney R, Porter J, Drobniewski F. Evaluation of a new rapid bacteriophage-based method for the drug susceptibility testing of Mycobacterium tuberculosis. Nature Medicine 1997; 3: 465-468.

40. David HL. Probability distribution of drug-resistant mutants in unselected populations of Mycobacterium tuberculosis. Appl Microbiol 1970; 20: 810-814. 